\title{
Ganho ponderal e estado nutricional de mulheres portadoras de diabetes mellitus
}

\section{gestacional}

\author{
Weight gain and nutritional status of women with gestational diabetes mellitus \\ Ganancia de peso y estado nutricional de mujeres portadoras de diabetes mellitus gestacional
}

\section{Resumo}

Objetivo: Analisar o ganho ponderal e o estado nutricional de gestantes portadoras de diabetes mellitus gestacional. Metodologia: Trata-se de um estudo de caráter descritivo, com corte transversal, realizado entre agosto a dezembro de 2020, em serviço de referência de gestação de alto risco de Pernambuco. Foram estudadas variáveis antropométricas, sociodemográficas, clínicas, obstétricas e de estilo de vida. Para análise estatística foram utilizados os testes QuiQuadrado de Pearson e Exato de fisher, quando aplicável, com nível de significância de 5\%. Resultados: Foram avaliadas 101 gestantes com idade de 30,73 \pm 6,29 anos, a maioria $(79,2 \%)$ com excesso de peso prévio e com elevação durante a gestação $(85,1 \%)$. O ganho ponderal acima do recomendado foi predominante $(45,3 \%)$, revelando associação positiva com o estado nutricional atual. A presença de patologias associadas ao DMG foi a única variável obstétrica, que apresentou associação significativa com o estado nutricional prévio, atual e ganho ponderal gestacional, independente do tipo de comorbidades. Conclusão: $\mathrm{O}$ ganho ponderal acima do recomendado foi predominante, assim como a frequência de excesso de peso antes e durante a gestação, o que evidencia a importância do acompanhamento nutricional em portadoras de diabetes mellitus gestacional.

Palavras-chave: Diabetes gestacional; Ganho de peso na gestação; Estado nutricional.

\begin{abstract}
Objective: To analyze weight gain and nutritional status of pregnant women with diabetes mellitus gestational. Methodology: This is a descriptive, cross-sectional study, conducted from August to December 2020, in a high-risk pregnancy reference service in Pernambuco. Information on anthropometric, socioeconomic, demographic, clinical, obstetric and lifestyle data were collected. To statistical analysis the Pearson's Chi-Square and Fisher's Exact tests,
\end{abstract}


were performed, when applicable, at 5\% significance level. Results: 101 high-risk pregnant women were studied, with mean age $30,73 \pm 6,29$, most $(79,2 \%)$ with previous overweight and with weight gain during gestation $(85,1 \%)$. Excessive weight gain was prevalent $(45,3 \%)$, revealing positive association with current nutritional status. The presence of pathologies associated with DMG was the only obstetric data which showed a significative association with previous and current nutritional status and gestational weight gain, regardless of the type of comorbidities. Conclusion: Excess weight gain was prevalent, as well as the frequency of overweight before and during pregnancy, which shows the importance of nutritional monitoring in patients with gestational diabetes mellitus.

Keywords: Diabetes gestational; Gestational weight gain; Nutritional status.

\section{Resumen}

Objetivo: Analizar la ganancia de peso y el estado nutricional de gestantes portadoras de diabetes mellitus gestacional. Metodología: Se trata de un estudio de carácter descriptivo, corte transversal, realizado entre agosto y diciembre de 2020, en un servicio de referencia de gestación de alto riesgo de Pernambuco. Se estudiaron variables antropométricas, sociodemográficas, clínicas, obstétricas y de estilo de vida. Para el análisis estadístico fueron utilizadas las pruebas Chi-cuadrada de Pearson o Exacta de Fisher, cuando aplicable, con un nivel de significación del 5\%. Resultados: Fueron evaluadas 101 gestantes con edad de 30,73 $\pm 6,29$ años, la mayoría $(79,2 \%)$ con exceso de peso y con elevación durante el embarazo $(85,1 \%)$. El aumento de peso acima de las recomendaciones fue predominante $(45,3 \%)$ revelando asociación positiva con el estado nutricional actual. La presencia de patologías asociadas al DMG fue la única variable obstétrica que presentó asociación significativa con el estado nutricional pregravídico, actual y ganancia de peso gestacional, independientemente del tipo de comorbilidades. Conclusión: El aumento de peso acima de las recomendaciones fue predominante así como la frecuencia de exceso de peso antes y durante la gestación, lo que evidencia la importancia del acompañamiento nutricional en mujeres portadoras de diabetes mellitus gestacional.

Palabras clave: Diabetes gestacional; Ganancia de peso gestacional; Estado nutricional.

\section{Introdução}

A gestação é um período de intensas mudanças fisiológicas, no qual ocorrem adaptações para garantir o suprimento adequado de nutrientes, bem como condições para o adequado crescimento e desenvolvimento fetal (Barbosa et al., 2013). A hiperinsulinemia e resistência à insulina são adaptações que ocorrem com frequência nesse período, estando relacionadas à complicações como o desenvolvimento de diabetes mellitus gestacional (DMG) (Plows et al., 2018).

O DMG é considerado o distúrbio metabólico mais comum na gestação, caracterizando-se por alteração no metabolismo dos carboidratos que se inicia durante a gestação, podendo persistir ou não após o parto (Sociedade Brasileira de Diabetes [SBD], 2019). Sua prevalência a nível mundial varia entre $1 \%$ a $28 \%$, a depender dos critérios diagnósticos e características da população (Behboudi-Gandevani et al., 2019; Jiwani et al., 2011). Enquanto no Brasil estima-se que as taxas estejam em torno de $18 \%$ no SUS (Trujillo, 2014).

A fisiopatologia desta doença está relacionada com a elevação de hormônios contrarreguladores da insulina, que ocorre devido ao estresse fisiológico imposto pela gravidez, além de fatores predeterminantes genéticos e ambientais (Johns et al., 2018; Law \& Zhang, 2017). O principal hormônio relacionado com a redução da sensibilidade e resistência à insulina é o lactogênio placentário, contudo, sabe-se que outros hormônios hiperglicemiantes como cortisol, estrógeno, progesterona e prolactina também estão envolvidos (Alfadhli, 2015).

O diagnóstico pode ser realizado por meio de testes provocativos com sobrecarga de glicose (teste oral de tolerância à glicose - TOTG), medição de glicose no plasma (após jejum mínimo de 8 horas) a partir do segundo trimestre da gestação e triagem precoce de gestantes de alto risco na primeira consulta pré-natal (American Diabetes Association, 2020).

Visto como uma das principais causas de morbimortalidade materna, o DMG está associado a síndromes hipertensivas na gestação - SHG (hipertensão, pré-eclâmpsia e eclâmpsia), aumento das taxas de cesariana, macrossomia e hiperinsulinemia fetal (AshwaL \& Hod, 2015; Huet et al., 2018). Ao mesmo tempo, é relacionado à problemas a longo prazo como diabetes mellitus tipo 2 (DM2), síndrome metabólica e doença cardiovascular (Cianni et al., 2018; Kautzky-Willer et al., 2019; Shen et al., 2019). 
Dentre os principais fatores de risco destacam-se: idade avançada, história familiar de DM2 em parentes de primeiro grau, síndrome de ovários policísticos e baixa estatura (Ribeiro et al., 2015; Zaman et al., 2018). Além destes, são citados na literatura, excesso de peso e ganho ponderal acima do recomendado (Braz et al., 2016; Djaković et al., 2019; Gou et al., 2019; Hantoushzadeh et al., 2016).

Estudos evidenciam importante associação entre obesidade e DMG afirmando que o ganho ponderal excessivo na gravidez atual e deposição central excessiva de gordura corporal estão relacionados a maiores níveis de glicemia e maior resistência à insulina (Martínez-Hortelano et al., 2019; Sorbye et al., 2017).

Gao et al. (2017) observaram que o sobrepeso, a obesidade e a adiposidade central contribuem com o aumento do risco de DMG. Achados que corroboram com o estudo de Martin et al. (2015), no qual mulheres que iniciaram a gestação com excesso de peso, apresentaram maior risco de desenvolver DMG, à medida que houve aumento do índice de massa corporal (IMC).

As intervenções nutricionais e a prática regular de atividade física (desde que sejam respeitadas as contraindicações obstétricas) são fundamentais no manejo do DMG. A orientação alimentar objetiva contribuir para um ganho ponderal adequado, bem como o bom controle metabólico. O tratamento atual também consiste no uso de farmacoterapia quando necessário e no monitoramento das glicemias capilares pré e pós-prandiais (SBD, 2019).

Assim, ao considerar que o DMG constitui um relevante problema de saúde pública, o qual está associado a piores desfechos durante o ciclo gravídico-puerperal, o presente estudo objetivou analisar o ganho ponderal e o estado nutricional de gestantes portadoras de DMG, cujos resultados podem subsidiar a atualização de condutas nutricionais para o tratamento e manejo dessa patologia.

\section{Metodologia}

Trata-se de um estudo de caráter descritivo, com corte transversal, realizado entre agosto e dezembro de $2020 \mathrm{em}$ um serviço de referência de gestação de alto risco de Pernambuco. Foram avaliadas gestantes, internadas nas enfermarias do centro obstétrico (COB), maternidade ou acompanhadas no pré-natal de alto risco do Hospital das Clínicas da Universidade Federal de Pernambuco (HC-UFPE).

A seleção da amostra foi realizada de forma não probabilística por conveniência, sendo incluídas de acordo com os seguintes critérios de elegibilidade: gestantes diagnosticadas com DMG, que estavam no segundo ou terceiro trimestre, com idade igual ou acima de 19 anos. Foram excluídas aquelas sem condições de responder o questionário da pesquisa (com problemas psiquiátricos e/ou cognitivos), que apresentavam gestação múltipla, ou insuficiência renal crônica, hepatopatias, cardiopatias graves, além daquelas que tinham realizado cirurgia plástica ou bariátrica, motivos que poderiam alterar a composição corporal.

A pesquisa foi realizada após aprovação pelo Comité de Ética em Pesquisa do HC/UFPE, sob o CAEE 26966719.3.0000.8807, em obediência à Resolução 466/12, do Conselho Nacional de Saúde do Ministério da Saúde.

A coleta de dados foi iniciada após assinatura de Termo de Consentimento Livre e Esclarecido (TCLE), sendo a caracterização da amostra realizada em questionário estruturado contendo informações obtidas através de entrevista direta, consultas ao prontuário e cartão da gestante. Vale ressaltar que medidas foram tomadas a fim de garantir a segurança dos envolvidos durante a realização da entrevista, entre elas, o distanciamento de 1,5 metros e a utilização de equipamentos de proteção individual (EPIs) como preconizado pelo Ministério da Saúde.

Foram consideradas variáveis sociodemográficas (idade, raça, estado civil, escolaridade, ocupação, renda familiar e procedência), de estilo de vida (atividade física prévia e atual, tabagismo e alcoolismo antes e durante a gestação), clínicas (tipo de tratamento e de patologias associadas, história prévia de DMG e história familiar de DM2), obstétricas (trimestre 
gestacional, número de gestações, número de partos, presença de abortamento), e antropométricas (peso pré-gestacional, peso atual, IMC prévio e atual e o ganho ponderal).

O estado nutricional prévio foi determinado através do cálculo do IMC, utilizando o peso pré-gestacional em quilos, dividido pela altura em metros ao quadrado $\left(\mathrm{kg} / \mathrm{m}^{2}\right)$, conforme recomendações da Organização Mundial da Saúde (2000). Foi considerado baixo peso o IMC $\leq 18,4 \mathrm{~kg} / \mathrm{m}^{2}$, eutrofia de 18,5 a $24,9 \mathrm{~kg} / \mathrm{m}^{2}$ e excesso de peso se igual ou superior a $25 \mathrm{~kg} / \mathrm{m}^{2}$. Também foi investigado o ganho ponderal e classificado pelo IMC pré-gestacional, utilizando-se as faixas recomendadas pelo Institute of Medicine (2009). O estado nutricional gestacional foi obtido através por meio do IMC, utilizando o peso atual dividido pela altura em metros ao quadrado $\left(\mathrm{kg} / \mathrm{m}^{2}\right)$ e classificado de acordo com a idade gestacional, conforme o gráfico de Atalah et al. (1997).

A análise dos dados foi realizada por meio de técnicas de estatística descritiva e inferencial. Os resultados descritivos foram expressos por meio de frequências absolutas e percentuais para as variáveis categóricas e em medidas: média e desvio padrão (média \pm DP) para as variáveis numéricas. Na avaliação de diferença entre os percentuais relativos às categorias de uma variável foi utilizado o teste Qui-quadrado de Pearson para igualdade de proporções em uma amostra. No estudo da associação entre duas variáveis categóricas foi utilizado o teste Qui-quadrado de Pearson ou o Exato de Fisher, quando a condição para utilização do teste Qui-quadrado não foi verificada.

O nível de significância utilizado na decisão dos testes estatísticos foi de 5\%. Os intervalos foram obtidos com $95 \%$ de confiança. Os dados foram digitados em planilha EXCEL e o programa utilizado para obtenção dos cálculos estatísticos foi o IMB SPSS na versão 25.0.

\section{Resultados}

Foram avaliadas 101 gestantes com DMG, com idade média de 30,73 $\pm 6,29$ anos e predominância da faixa etária de 30-34 anos (31,7\%), procedentes da Região Metropolitana do Recife (60,4\%), não brancas $(82,2 \%)$, casadas/união estável $(67,3 \%)$, com mais de oito anos de estudo $(66,3 \%)$, desempregadas $(59,4 \%)$ e com renda familiar inferior a um salário mínimo $(64,4 \%)$ (Tabela 1$)$.

Quanto ao estilo de vida, 68,3\% eram sedentárias/inativas previamente e durante a gestação o percentual aumentou para $89,1 \%$, possivelmente devido as medidas de restrição e isolamento social impostas pela atual situação de pandemia. Em relação à ingestão de bebidas alcóolicas, 67,3\% eram abstêmicas previamente, percentual que foi ainda maior durante a gestação (98\%). Enquanto 14,9\% afirmaram tabagismo prévio, 3\% mantiveram o hábito durante o período gestacional (Tabela 1). 
Tabela 1 - Características sociodemográficas e de estilo de vida, de mulheres portadoras de diabetes mellitus gestacional. HC/UFPE, Recife-PE, 2020.

\begin{tabular}{|c|c|c|c|}
\hline Variável & $\mathbf{N}$ & $\%$ & Valor de $\mathbf{P}$ \\
\hline Grupo Total & 101 & 100 & \\
\hline Faixa etária & & & $\mathrm{p}^{(1)}=0,009^{*}$ \\
\hline 19 a 24 & 21 & 20,8 & \\
\hline 25 a 29 & 21 & 20,8 & \\
\hline 30 a 34 & 32 & 31,7 & \\
\hline 35 a 45 & 27 & 26,7 & \\
\hline Raça & & & $\mathrm{p}^{(1)}<0,001^{*}$ \\
\hline Branca & 18 & 17,8 & \\
\hline Não branca & 83 & 82,2 & \\
\hline Estado civil & & & $\mathrm{p}^{(1)}<0,001^{*}$ \\
\hline Casada/ União estável & 68 & 67,3 & \\
\hline Solteira/ Divorciada/ Viúva & 33 & 32,7 & \\
\hline Escolaridade & & & $\mathrm{p}^{(1)}=0,001^{*}$ \\
\hline$\geq 8$ anos de estudos & 67 & 66,3 & \\
\hline$<8$ anos de estudos & 34 & 33,7 & \\
\hline Ocupação & & & $\mathrm{p}^{(1)}=0,059^{*}$ \\
\hline Trabalha & 41 & 40,6 & \\
\hline Não trabalha & 60 & 59,4 & \\
\hline Renda familiar (2) & & & $\mathrm{p}^{(1)}=0,004^{*}$ \\
\hline$\geq 1$ salário & 36 & 35,6 & \\
\hline$<1$ salário & 65 & 64,4 & \\
\hline Procedência & & & $\mathrm{p}^{(1)}<0,001^{*}$ \\
\hline Região Metropolitana do Recife & 61 & 60,4 & \\
\hline Interior/ Outros & 40 & 39,6 & \\
\hline Atividade física pré-gestacional & & & $\mathrm{p}^{(1)}<0,001 *$ \\
\hline Sim & 32 & 31,7 & \\
\hline Não & 69 & 68,3 & \\
\hline Atividade física gestacional & & & $\mathrm{p}^{(1)}<0,001^{*}$ \\
\hline Sim & 11 & 10,9 & \\
\hline Não & 90 & 89,1 & \\
\hline Tabagismo pré-gestacional & & & $\mathrm{p}^{(1)}<0,001 *$ \\
\hline Sim & 15 & 14,9 & \\
\hline Não & 86 & 85,1 & \\
\hline Tabagismo gestacional & & & $\mathrm{p}^{(1)}<0,001 *$ \\
\hline Sim & 3 & 3 & \\
\hline Não & 98 & 97 & \\
\hline Etilismo pré-gestacional & & & $\mathrm{p}^{(1)}<0,001^{*}$ \\
\hline Sim & 33 & 32,7 & \\
\hline Não & 68 & 67,3 & \\
\hline Etilismo gestacional & & & $\mathrm{p}^{(1)}<0,001 *$ \\
\hline Sim & 2 & 2 & \\
\hline Não & 99 & 98 & \\
\hline
\end{tabular}

Legenda: (*) Diferença significativa ao nível de 5,0\%. (1) Pelo teste Qui-quadrado para uma amostra. (2) Valor da renda em $2020.2=\mathrm{R} \$ 1.045$. Fonte: Autores. 
Research, Society and Development, v. 10, n. 3, e48510313565, 2021

(CC BY 4.0) | ISSN 2525-3409 | DOI: http://dx.doi.org/10.33448/rsd-v10i3.13565

Em relação às variáveis clínicas e obstétricas, predominou o terceiro trimestre gestacional e a falta de história prévia de DMG. A presença de patologias associadas esteve presente na maioria do grupo, a qual se destacou as síndromes hipertensivas (Tabela 2).

Quando associadas ao estado nutricional pré-gestacional, apenas a variável patologia mostrou significância estatística, com a maioria das gestantes apresentando excesso de peso pré gestacional (Tabela 2). 
Tabela 2 - Estado nutricional pré-gestacional de acordo com variáveis clínicas e obstétricas de mulheres portadoras de diabetes mellitus gestacional. HC/UFPE, Recife-PE, 2020.

\section{IMC pré-gestacional}

Variável

Grupo Total

Trimestre gestacional

Segundo

Terceiro

$5(5)$
$96(95)$

Número de gestações

Primigesta

Multigesta

$23(22,8)$

$78(77,2)$

Número de partos

Nulípara

Primípara

Multípara

$27(26,7)$
$34(33,7)$
$40(39,6)$

$65(64,4)$

$36(35,6)$

$43(42,6)$

DMG exclusivo

DMG e SHG

DMG e outras comorbidades

Tratamento para DMG

Sem tratamento

Dieta

Hipoglicemiante oral/Insulina

História prévia de DMG

Sim

Não

$10(9,9)$

$30(29,7)$

$67(66,3)$

4 (4)

$18(66,7)$

$26(76,5)$

$36(90,0)$

$52(80,0)$

$28(77,8)$

$30(69,8)$

$39(90,7)$

$11(73,3)$

$26(86,7)$

$51(76,1)$

$3(75,0)$

$91(90,1)$

História familiar de DM

Sim

$61(60,4)$

$40(39,6)$
$8(80,0)$

$72(79,1)$

$47(77,0)$

$33(82,5)$
Não

$$
\mathrm{p}^{(1)}=0,581
$$

$21(21,9)$

$6(26,1)$

$15(19,2)$

$9(33,3)$

$8(23,5)$

$4(10,0)$

$$
\mathrm{p}^{(1)}=0,560
$$

$$
\mathrm{p}^{(2)}=0,062
$$

$13(20,0)$

$8(22,2)$

$13(30,2)$

$4(9,3)$

$4(26,7)$

$$
\mathrm{p}^{(2)}=0,792
$$

$4(13,3)$

$16(23,9)$

$1(25,0)$

$$
\mathrm{p}^{(1)}=0,042 *
$$

$2(20,0)$

$19(20,9)$

$$
\mathrm{p}^{(2)}=1,000
$$

$14(23,0)$

$\mathrm{p}^{(2)}=0,509$

Legenda: IMC: índice de massa corporal. SHG: síndromes hipertensivas na gestação. DMG: diabetes mellitus gestacional. DM: diabetes mellitus. (*) Diferença significativa ao nível de 5,0\%. (1) Pelo teste Qui-quadrado de Pearson. (2) Pelo teste Exato de Fisher. Fonte: Autores.

Não foi observada associação estatística, entre o estado nutricional atual e variáveis clínicas e obstétricas, com exceção da presença de patologias associadas, sendo constatada maior frequência de excesso de peso gestacional naquelas com DMG e SHG, e com DMG e outras comorbidades (Tabela 3). 
Tabela 3 - Estado nutricional atual de acordo com variáveis clínicas e obstétricas de mulheres portadoras de diabetes mellitus gestacional. HC/UFPE, Recife-PE, 2020.

\begin{tabular}{|c|c|c|c|c|}
\hline \multirow[b]{2}{*}{ Variável } & \multicolumn{4}{|c|}{ IMC atual } \\
\hline & $\begin{array}{c}\text { TOTAL } \\
\text { n }(\%)\end{array}$ & $\begin{array}{c}\text { Com excesso de peso } \\
\mathrm{n}(\%)\end{array}$ & $\begin{array}{c}\text { Sem excesso de peso } \\
\mathrm{n}(\%)\end{array}$ & Valor de $p$ \\
\hline Grupo Total & & $86(85,1)$ & $15(14,9)$ & \\
\hline Trimestre gestacional & & & & $\mathrm{p}^{(2)}=0,560$ \\
\hline Segundo & $5(5)$ & $4(80,0)$ & $1(20,0)$ & \\
\hline Terceiro & $96(95)$ & $82(85,4)$ & $14(14,6)$ & \\
\hline Número de gestações & & & & $\mathrm{p}^{(2)}=0,741$ \\
\hline Primigesta & $23(22,8)$ & $19(82,6)$ & $4(17,4)$ & \\
\hline Multigesta & $78(77,2)$ & $67(85,9)$ & $11(14,1)$ & \\
\hline Número de partos & & & & $\mathrm{p}^{(1)}=0,068$ \\
\hline Nulípara & $27(26,7)$ & $22(81,5)$ & $5(18,5)$ & \\
\hline Primípara & $34(33,7)$ & $26(76,5)$ & $8(23,5)$ & \\
\hline Multípara & $40(39,6)$ & $38(95,0)$ & $2(5,0)$ & \\
\hline Número de abortos & & & & $\mathrm{p}^{(1)}=0,334$ \\
\hline Nenhum & $65(64,4)$ & $57(87,7)$ & $8(12,3)$ & \\
\hline Um ou mais & $36(35,6)$ & $29(80,6)$ & $7(19,4)$ & \\
\hline Patologia & & & & $\mathrm{p}^{(1)}=0,033^{*}$ \\
\hline DMG exclusivo & $43(42,6)$ & $32(74,4)$ & $11(25,6)$ & \\
\hline DMG e SHG & $43(42,6)$ & $40(93,0)$ & $3(7,0)$ & \\
\hline DMG e outras comorbidades & $15(14,9)$ & $14(93,3)$ & $1(6,7)$ & \\
\hline Tratamento para DMG & & & & $\mathrm{p}^{(2)}=0,352$ \\
\hline Sem tratamento & $30(29,7)$ & $24(80,0)$ & $6(20,0)$ & \\
\hline Dieta & $67(66,3)$ & $59(88,1)$ & $8(11,9)$ & \\
\hline Hipoglicemiante oral/Insulina & $4(4)$ & $3(75,0)$ & $1(25,0)$ & \\
\hline História prévia de DMG & & & & $\mathrm{p}^{(2)}=0,167$ \\
\hline Sim & $10(9,9)$ & $7(70,0)$ & $3(30,0)$ & \\
\hline Não & $91(90,1)$ & $79(86,8)$ & $12(13,2)$ & \\
\hline História familiar de DM & & & & $\mathrm{p}^{(1)}=0,267$ \\
\hline Sim & $61(60,4)$ & $50(82,0)$ & $11(18,0)$ & \\
\hline Não & $40(39,6)$ & $36(90,0)$ & $4(10,0)$ & \\
\hline
\end{tabular}

Legenda: IMC: índice de massa corporal. SH: síndromes hipertensivas na gestação. DMG: diabetes mellitus gestacional. DM: diabetes mellitus. (*) Diferença significativa ao nível de 5,0\%. (1) Pelo teste Qui-quadrado de Pearson. (2) Pelo teste Exato de Fisher. Fonte: Autores.

Ao relacionar o ganho ponderal gestacional com variáveis clínicas e obstétricas, apenas a variável patologia evidenciou associação, prevalecendo o ganho ponderal excessivo, independentemente da presença ou não de comorbidades relacionadas ao DMG (Tabela 4). 
Tabela 4 - Ganho ponderal gestacional de acordo com variáveis clínicas e obstétricas de mulheres portadoras de diabetes mellitus gestacional. HC/UFPE, Recife-PE, 2020.

\section{Ganho ponderal gestacional}

\begin{tabular}{|c|c|c|c|c|c|}
\hline Variável & $\begin{array}{c}\text { TOTAL } \\
\mathrm{n}(\%)\end{array}$ & $\begin{array}{c}\text { Insuficiente } \\
\mathrm{n}(\%)\end{array}$ & $\begin{array}{c}\text { Adequado } \\
\text { n (\%) }\end{array}$ & $\begin{array}{c}\text { Excessivo } \\
\mathrm{n}(\%)\end{array}$ & Valor de p \\
\hline Grupo Total & & $21(22,6)$ & $28(30,1)$ & $44(47,3)$ & \\
\hline $\begin{array}{l}\text { Trimestre gestacional } \\
\text { Segundo } \\
\text { Terceiro }\end{array}$ & $\begin{array}{c}5(5) \\
96(95)\end{array}$ & $\begin{array}{c}1(33,3) \\
20(22,2)\end{array}$ & $28(31,1)$ & $\begin{array}{c}2(66,7) \\
42(46,7)\end{array}$ & $\mathrm{p}^{(2)}=0,597$ \\
\hline $\begin{array}{l}\text { Número de gestações } \\
\text { Primigesta } \\
\text { Multigesta }\end{array}$ & $\begin{array}{l}23(22,8) \\
78(77,2)\end{array}$ & $\begin{array}{c}5(23,8) \\
16(22,2)\end{array}$ & $\begin{array}{c}4(19,0) \\
24(33,3)\end{array}$ & $\begin{array}{l}12(57,1) \\
32(44,4)\end{array}$ & $\mathrm{p}^{(1)}=0,433$ \\
\hline $\begin{array}{l}\text { Número de partos } \\
\text { Nulípara } \\
\text { Primípara } \\
\text { Multípara }\end{array}$ & $\begin{array}{l}27(26,7) \\
34(33,7) \\
40(39,6)\end{array}$ & $\begin{array}{l}6(24,0) \\
7(22,6) \\
8(21,6)\end{array}$ & $\begin{array}{l}5(20,0) \\
12(38,7) \\
11(29,7)\end{array}$ & $\begin{array}{l}14(56,0) \\
12(38,7) \\
18(48,6)\end{array}$ & $\mathrm{p}^{(1)}=0,636$ \\
\hline $\begin{array}{l}\text { Aborto } \\
\text { Não } \\
\text { Sim }\end{array}$ & $\begin{array}{l}65(64,4) \\
36(35,6)\end{array}$ & $\begin{array}{l}10(16,4) \\
11(34,4)\end{array}$ & $\begin{array}{c}20(32,8) \\
8(25,0)\end{array}$ & $\begin{array}{l}31(50,8) \\
13(40,6)\end{array}$ & $\mathrm{p}^{(1)}=0,143$ \\
\hline $\begin{array}{l}\text { Patologia } \\
\text { DMG exclusivo } \\
\text { DMG e SHG } \\
\text { DMG e outras comorbidades }\end{array}$ & $\begin{array}{l}43(42,6) \\
43(42,6) \\
15(14,9)\end{array}$ & $\begin{array}{c}13(32,5) \\
3(7,7) \\
5(35,7)\end{array}$ & $\begin{array}{l}10(25,0) \\
15(38,5) \\
3(21,4)\end{array}$ & $\begin{array}{c}17(42,5) \\
21(53,8) \\
6(42,9)\end{array}$ & $\mathrm{p}^{(2)}=0,048^{*}$ \\
\hline $\begin{array}{l}\text { Tratamento para DMG } \\
\text { Sem tratamento } \\
\text { Dieta } \\
\text { Hipoglicemiante oral/Insulina }\end{array}$ & $\begin{array}{c}30(29,7) \\
67(66,3) \\
4(4)\end{array}$ & $\begin{array}{c}7(25,0) \\
12(19,7) \\
2(50,0)\end{array}$ & $\begin{array}{l}10(35,7) \\
18(29,5) \\
\quad-\end{array}$ & $\begin{array}{l}11(39,3) \\
31(50,8) \\
2(50,0)\end{array}$ & $\mathrm{p}^{(2)}=0,450$ \\
\hline $\begin{array}{l}\text { História prévia de DMG } \\
\text { Sim } \\
\text { Não }\end{array}$ & $\begin{array}{c}10(9,9) \\
91(90,1)\end{array}$ & $\begin{array}{c}1(12,5) \\
20(23,5)\end{array}$ & $\begin{array}{c}3(37,5) \\
25(29,4)\end{array}$ & $\begin{array}{c}4(50,0) \\
40(47,1)\end{array}$ & $\mathrm{p}^{(2)}=0,894$ \\
\hline $\begin{array}{l}\text { História familiar de DM } \\
\text { Sim } \\
\text { Não }\end{array}$ & $\begin{array}{l}61(60,4) \\
40(39,6)\end{array}$ & $\begin{array}{c}14(25,5) \\
7(18,4)\end{array}$ & $\begin{array}{c}20(36,4) \\
8(21,1)\end{array}$ & $\begin{array}{l}21(38,2) \\
23(60,5)\end{array}$ & $\mathrm{p}^{(1)}=0,100$ \\
\hline
\end{tabular}

Legenda: SH: síndromes hipertensivas na gestação. DMG: diabetes mellitus gestacional. DM: diabetes mellitus. (*) Diferença significativa ao nível de 5,0\%. (1) Pelo teste Qui-quadrado de Pearson. (2) Pelo teste Exato de Fisher. Fonte: Autores.

No que se refere às variáveis antropométricas, observou-se que o IMC pré-gestacional foi de $30,12 \pm 6,42 \mathrm{~kg} / \mathrm{m}^{2} \mathrm{e}$ $79,2 \%$ da amostra se encontrava acima do peso. Durante o período gestacional o IMC foi de $34,16 \pm 5,94 \mathrm{~kg} / \mathrm{m}^{2}, \mathrm{com}$ excesso de peso em $85,1 \%$ do grupo, sendo detectado ganho ponderal acima do recomendado em 47,3\%, com média de ganho ponderal de $11,61 \pm 6,28 \mathrm{~kg}$; levando-se em consideração o número amostral de 93 gestantes, uma vez que 8 apresentaram perda de peso gestacional.

Conforme apresentado na Tabela 5, na associação do ganho ponderal gestacional com o estado nutricional prévio a gestação e com o estado nutricional gestacional, houve associação estatística com o atual. Foi detectado que 64,3\% das 
mulheres sem excesso de peso na gestação, apresentaram ganho ponderal insuficiente, enquanto aquelas com excesso de peso obtiveram um ganho predominantemente excessivo entre $43,3 \%$ a $61,2 \%$.

Tabela 5 - Avaliação do ganho ponderal gestacional segundo o IMC pré-gestacional e IMC atual de mulheres portadoras de diabetes mellitus gestacional. HC/UFPE, Recife-PE, 2020.

\begin{tabular}{|c|c|c|c|c|c|}
\hline \multirow[b]{2}{*}{ Variável } & \multicolumn{5}{|c|}{ Ganho ponderal gestacional } \\
\hline & $\begin{array}{c}\text { TOTAL } \\
\mathrm{n}(\%)\end{array}$ & $\begin{array}{c}\text { Insuficiente } \\
\mathrm{n}(\%)\end{array}$ & $\begin{array}{c}\text { Adequado } \\
\mathrm{n}(\%)\end{array}$ & $\begin{array}{c}\text { Excessivo } \\
\mathrm{n}(\%)\end{array}$ & Valor de $p$ \\
\hline Grupo Total & $93(100)$ & $21(22,6)$ & $28(30,1)$ & $44(47,3)$ & \\
\hline $\begin{array}{l}\text { IMC pré-gestacional } \\
\text { Sem excesso } \\
\text { Sobrepeso } \\
\text { Obesidade }\end{array}$ & $\begin{array}{l}21(22,6) \\
34(36,5) \\
38(40,9)\end{array}$ & $\begin{array}{l}6(28,6) \\
8(23,5) \\
7(18,4)\end{array}$ & $\begin{array}{c}6(28,6) \\
8(23,5) \\
14(36,8)\end{array}$ & $\begin{array}{c}9(42,9) \\
18(52,9) \\
17(44,7)\end{array}$ & $\mathrm{p}^{(1)}=0,720$ \\
\hline $\begin{array}{l}\text { IMC gestacional } \\
\text { Sem excesso } \\
\text { Sobrepeso } \\
\text { Obesidade }\end{array}$ & $\begin{array}{c}14(15) \\
30(32,3) \\
49(52,7)\end{array}$ & $\begin{array}{l}9(64,3) \\
6(20,0) \\
6(12,2)\end{array}$ & $\begin{array}{l}4(28,6) \\
11(36,7) \\
13(26,5)\end{array}$ & $\begin{array}{c}1(7,1) \\
13(43,3) \\
30(61,2)\end{array}$ & $\mathrm{p}^{(2)}=0,001^{*}$ \\
\hline
\end{tabular}

Legenda: IMC: índice de massa corporal. (*) Diferença significativa ao nível de 5,0\%. (1) Pelo teste Qui-quadrado de Pearson. (2) Pelo teste Exato de Fisher. Fonte: Autores.

\section{Discussão}

O ganho ponderal excessivo e a obesidade são fatores relacionados ao aumento de complicações materno-fetais em gestantes com DMG, principalmente naquelas que apresentam fatores de risco associados a patologia, como histórico sociodemográfico, clínico e obstétrico.

De acordo com a literatura, o perfil sociodemográfico pode influenciar diretamente os resultados e a qualidade de vida de gestantes (Iwanowicz-Palus et al., 2020). Pesquisas no Sudeste Brasileiro em usuárias do SUS encontraram resultados semelhantes ao do presente estudo. Abreu et al. (2019) encontraram em portadoras de DMG, maior índice de desemprego (55,6\%) e renda inferior a um salário mínimo (81\%). Enquanto Silva et al. (2018) detectaram mulheres com idade de 31,2 anos, não brancas (64,4\%), em união estável (86,4\%) e com ensino médio (76\%).

Galliano et al. (2018) ao avaliarem gestantes com DMG em três cidades brasileiras, detectaram inatividade física gestacional expressiva $(65,7 \%)$, diferente do tabagismo que não foi predominante $(24,7 \%)$, assim como ocorreu no presente estudo. Enquanto outra pesquisa realizada na cidade do Recife/PE, constituída por 87\% de gestantes com DMG, evidenciou que pouco mais da metade $(50,9 \%)$ apresentou sedentarismo, sendo menor $(3,5 \%)$ o percentual de tabagismo e de etilismo $(1,8 \%)$ prévios (Cysneiros et al., 2020).

Quanto as variáveis clínicas e obstétricas, estudo no Nordeste Brasileiro identificou maior percentual de gestantes com DMG no terceiro trimestre, multigestas (82\%), menor frequência de abortos (26\%), elevada hereditariedade para diabetes (44\%) e dieta como única forma de tratamento (60\%), coincidindo com resultados deste grupo. Do mesmo modo em que essas variáveis não foram associadas ao IMC pré-gestacional (Queiroz et al., 2016). Dados de Yen et al. (2019) em estudo realizado em Taiwan mostram resultados semelhantes, com menor história prévia de DMG (27,8\%).

No presente estudo a variável patologia esteve relacionada ao IMC e ao ganho ponderal, independente da presença ou do tipo de comorbidades associadas ao DMG, a maioria das pacientes apresentavam excesso de peso pré-gestacional. A 
literatura é escassa quanto a resultados que realizaram esta associação, nos impedindo de comparações, o que ressalta a necessidade de mais pesquisas que façam esta relação.

Pesquisas nacionais e internacionais com DMG, identificaram excesso de peso em período pré-gestacional, com variação de 47,8\% a 74\% (Guerra et al., 2018; Larrabure-Torrealva et al., 2018; Machado et al., 2019; Tavares et al., 2019). Configurando esta variável como importante fator de risco para DMG, entre mulheres em idade reprodutiva.

Quanto ao estado nutricional gestacional, outros autores também relataram excesso de peso na faixa de 63,9\% a 80\% dos grupos (Guerra et al., 2018; Nogueira et al., 2020), confirmando os resultados desta pesquisa, que ainda encontrou associação significativa desta variável com o ganho ponderal gestacional.

Em relação ao ganho ponderal excessivo, a literatura mostra variação de 26,1\% a 35,4\%, com maiores valores em mulheres com sobrepeso e obesidade pré-gestacional (36,7\% e 36,9\%) respectivamente. Ao mesmo tempo que gestantes de baixo peso $(48,3 \%)$ e eutróficas $(50,2 \%)$, podem apresentar ganho ponderal abaixo do recomendado (Machado et al., 2019; Tavares et al., 2019). Ademais foi observada associação estatística significante entre o ganho ponderal e o estado nutricional prévio no estudo de Machado et al. (2019); fato não observado no presente estudo.

Em estudo que comparou resultados de duas coortes multicêntricas brasileiras, com intervalo de quase 30 anos, foi identificado mudança no perfil nutricional das gestantes com DMG, com elevação do IMC pré-gestacional $\left(30,3 \pm 6,5 \mathrm{~kg} / \mathrm{m}^{2}\right.$ vs. $24,6 \pm 4,4 \mathrm{~kg} / \mathrm{m}^{2}$ ), prevalência de excesso de peso (79\% vs. $\left.43,1 \%\right)$ e ganho ponderal acima das recomendações $(35,7 \%$ vs. $27,3 \%$ ) na coorte mais recente, o que reflete o processo de transição nutricional vivenciado ao longo dos últimos anos no Brasil (Silveira et al., 2020).

Como limitações deste estudo, destacam-se a suspensão das pesquisas por longo período no início da pandemia causada pelo novo Coronavírus (SARS CoV2), as mudanças de comportamentos e hábitos de vida das gestantes com DMG, nesse período de confinamento e distanciamento, o que pode ter influenciado nas variáveis antropométricas e de estilo de vida. Além da redução do tamanho da amostra, aumentando a possibilidade de viés de informação.

\section{Conclusão}

Pode-se concluir que nessa amostra de gestantes portadoras de DMG, a frequência de excesso de peso foi verificada antes e durante a gestação, ao mesmo tempo que o ganho ponderal excessivo foi predominante, observando-se associação desta variável com o estado nutricional atual.

Esses achados corroboram com outros estudos nacionais e internacionais acerca da prevalência do excesso de peso e do ganho ponderal acima do recomendado nesta população, o que ressalta a importância do acompanhamento nutricional de gestantes com DMG e a necessidade de mais estudos, com amostras mais significativas, fora de períodos de confinamento, considerando a relevância e influência destes fatores nos desfechos materno-fetais.

\section{Referências}

Abreu, L. R. S., Shirley, M. K., Castro, N. P., Euclydes, V. V., Bergamaschi, D. P., Luzia, L. A., Cruz, A. M., \& Rondó, P. H. C. (2019). Gestational diabetes mellitus, pre-pregnancy body mass index, and gestational weight gain as risk factors for increased fat mass in Brazilian newborns. Plos One, 14(8), 221-971. https://doi.org/10.1371/journal.pone.0221971

Alfadhli, E. M. (2015). Gestational diabetes mellitus. Saudi Medical Journal, 36(4), 399-406. https://doi.org/10.15537/smj.2015.4.10307

American Diabetes Association. 2. (2020). Classification and diagnosis of diabetes: standards of medical care in diabetes - 2021. Diabetes Care, 44(1), 15-33. https://doi.org/10.2337/dc21-S002

Ashwal, E., \& Hod, M. (2015) Gestational diabetes mellitus: Where are we now? Clinica Chimica Acta, 451, 14-20. https://doi.org/10.1016/j.cca.2015.01.021

Atalah, E. S., Castillo, L. C., Castro S. R., \& Aldea P., A. (1997). Propuesta de um nuevo estándar de evaluación nutricional en embarazadas. Revista Médica del Chile, 125(12), 1429-1436. http://repositorio.uchile.cl/handle/2250/162816 
Barbosa, J. M., Neves, C. M. A. F., Araújo, L. L., \& Silva, E. M. C. (2013). Guia ambulatorial de nutrição materno-infantil. MedBook, p. 466.

Behboudi-Gandevani, S., Amiri, M., Yarandi, R. B., \& Tehrani, F. R. (2019). The impact of diagnostic criteria for gestational diabetes on its prevalence: a systematic review and meta-analysis. Diabetology Metabolic Syndrome, 11(1), 1-18. https://doi.org/10.1186/s13098-019-0406-1

Braz, L., Figueiredo, L., \& Fonseca, F. (2016). A influência da obesidade e ganho ponderal no peso do recém-nascido num grupo de grávidas com diabetes gestacional. Revista Portuguesa de Endocrinologia, Diabetes e Metabolismo, 8(2), 70-76. https://doi.org/10.1016/j.rpedm.2013.10.001

Cianni, G., Lacaria, E., Lencioni, C., \& Resi, V. (2018). Preventing type 2 diabetes and cardiovascular disease in women with gestational diabetes - The evidence and potential strategies. Diabetes Research And Clinical Practice, 145, 184-192. https://doi.org/10.1016/j.diabres.2018.04.021

Cysneiros, G. F., Nascimento, E., Araújo, E. C., Escoteiro, F. K. R. S., Paes-Silva, R. P., Barbosa, L. M. A., Burgos, M. G. P. A., \& Lemos, M. C. C. (2020). Estado nutricional e consumo alimentar de gestantes diabéticas atendidas em hospital de referência em Recife- PE. Brazilian Journal Of Development, 6(7), 46320-46335. https://doi.org/10.34117/bjdv6n7-311

Djaković, I., Soljačić-Vraneł, H., \& Kuna, K. (2019). Weight gain in pregnancy and weight retention after birth. Open Access Macedonian Journal Of Medical Sciences, 7(4), 614-616. https://doi.org/10.3889/oamjms.2019.141

Galliano, L. M., Vecchio, A. H. M., Silvani, J., \& Façanha, C. (2018). Physical activity level in women with gestational diabetes mellitus: lifestyle intervention for diabetes prevention after pregnancy (linda :brasil) study. Journal Of Diabetes, 11(6), 457-465. https://doi.org/10.1111/1753-0407.12872

Gao, X., Yan, Y., Xiang, S., Zeng, G., Liu, S., Sha, T., He, Q., Li, H., Tan, S., \& Chen, C. (2017). The mutual effect of pre-pregnancy body mass index, waist circumference and gestational weight gain on obesity-related adverse pregnancy outcomes: A birth cohort study. Plos One, 12(6), 177-418. https://doi.org/10.1371/journal.pone.0177418

Gou, B. H., Guan, H. M., Bi, Y. X., \& Ding, B. J. (2019). Weight gain during pregnancy and its relationship to pregnancy outcomes. Chinese Medical Journal, 132(2), 154-160. https://doi.org/10.1097/CM9.0000000000000036

Guerra, J. V. V., Alves, V. H., Rodrigues, D. P., Branco, M. B. L. R., Marchiori, G. R. S., \& Santos, M. V. (2018). Diabetes gestacional e estado nutricional materno em um hospital universitário de Niterói. Journal Nursing And Health, 8(8), 111-118. https://doi.org/10.15210/jonah.v8i1.13785

Hantoushzadeh, S., Sheikh, M., Bosaghzadeh, Z., Ghotbizadeh, F., Tarafdari, A., Panahi, Z., \& Shariat, M. (2016). The impact of gestational weight gain in different trimesters of pregnancy on glucose challenge test and gestational diabetes. Postgraduate Medical Journal, 92(1091), 520-524. https://doi.org/10.1136/postgradmedj-2015-133816

Huet, J., Beucher, G., Rod, A., Morello, R., \& Dreyfus, M. (2018) Joint impact of gestational diabetes and obesity on perinatal outcomes. Journal Of Gynecology Obstetrics And Human Reproduction, 47(9), 469-476. https://doi.org/10.1016 /j.jogoh.2018.08.003

Institute of Medicine. (2009). Weight gain during pregnancy: reexamining the guidelines. The National Academies Press. https://doi.org/10.17226/12584

Iwanowicz-Palus, G., Zarajczyk, M., \& Bien, A. (2020). The relationship between health-related quality of life, acceptance of illness and characteristics of pregnant women with hyperglycemia. Health And Quality Of Life Outcomes, 18(1), 319-325. https://doi.org/10.1186/s12955-020-01582-y

Jiwani, A., Marseille, E., Lohse, N., Damm, P., Hod, M., \& Kahn, J. G. (2011). Gestational diabetes mellitus: results from a survey of country prevalence and practices. The Journal Of Maternal-fetal \& Neonatal Medicine, 25(6), 600-610. https://doi.org/10.3109/14767058.2011.587921

Johns, E. C., Denison, F. C., Norman, J. E., \& Reynolds, R. M. (2018). Gestational diabetes mellitus: mechanisms, treatment, and complications. Trends In Endocrinology \& Metabolism, 29(11), 743-754. https://doi.org/10.1016/j.tem.2018.09.004

Kautzky-Willer, A., Harreiter, J., Winhofer-StöckL, Y., Bancher-Todesca, D., Berger, A., Repa, A., Lechleitner, M., \& Weitgasser, R. (2019). Gestations diabetes (GDM): update 2019. Wiener Klinische Wochenschrift, 131(1), 91-102. https://doi.org/10.1007/s00508-018-1419-8

Larrabure-Torrealva, G. T., Martinez, S., Fernandez, M. A. L., Sanchez, S. E., Mascaro, P. A., Ingar, H., Castillo, W., Zumaeta, R., Grande, M., \& Motta, V. (2018). Prevalence and risk factors of gestational diabetes mellitus: findings from a universal screening feasibility program in lima, peru. Bmc Pregnancy And Childbirth, 18(1), 303. https://doi.org/10.1186/s12884-018-1904-0

Law, K. P., \& Zhang, H. (2017). The pathogenesis and pathophysiology of gestational diabetes mellitus: deductions from a three-part longitudinal metabolomics study in China. Clinica Chimica Acta, 468, 60-70. https://doi.org/10.1016/j.cca.2017.02.008

Machado, C., Monteiro, S., \& Oliveira, M. J. (2019). Impact of overweight and obesity on pregnancy outcomes in women with gestational diabetes - results from a retrospective multicenter study. Archives Of Endocrinology And Metabolism, 64(1), 45-51. https://doi.org/10.20945/2359-3997000000178

Martin, K. E., Grivell, R. M., Yelland, L. N., \& Dodd, J. M. (2015). The influence of maternal BMI and gestational diabetes on pregnancy outcome. Diabetes Research And Clinical Practice, 108(3), 508-513. https://doi.org/10.1016/j.diabres.2014.12.015

Nogueira, M. D. A., Santos, C. C., Lima, A. M., Lima, M. R. S., Sousa, F. I. S., Vieira, L. C. O., Braga, R. A. M., \& Cruz, I. F. S. (2020). Associação entre estado nutricional, diabetes gestacional e doenças hipertensivas em gestantes de risco. Brazilian Journal Of Development, 6 (2), 8005-8018. https://doi:10.34117/bjdv6n2-20

Plows, J., Stanley, J., Baker, P., Reynolds, C., \& Vickers, M. (2018). The pathophysiology of gestational diabetes mellitus. International Journal Of Molecular Sciences, 19(11), 3342. https://doi.org/10.3390/ijms19113342

Queiroz, P. M. A., Souza, N. M. M., \& Burgos, M. G. P. A. (2016). Nutritional profile and associated factors in women with gestational diabetes. Nutrición Clínica y Dietetica Hospitalaria, 2, 96-102. https://doi:10.12873/362alburquerquequeiroz 
Research, Society and Development, v. 10, n. 3, e48510313565, 2021

(CC BY 4.0) | ISSN 2525-3409 | DOI: http://dx.doi.org/10.33448/rsd-v10i3.13565

Ribeiro, A. M. C., Silva, C. N., Rocha, G. M., \& Pereira, M. L. (2015). Diabetes gestacional: determinação de fatores de risco para diabetes mellitus. Revista Portuguesa de Endocrinologia, Diabetes e Metabolismo, 10(1), 8-13. https://doi.org/10.1016/j.rpedm.2014.05.004

Shen, Y., Li, W., Leng, J., Zhang, S., Liu, H., Li, W., Wang, L., Tian, H., Chen, J., \& Qi, L. (2019). High risk of metabolic syndrome after delivery in pregnancies complicated by gestational diabetes. Diabetes Research And Clinical Practice, 150, 219-226. https://doi.org/10.1016/j.diabres.2019.03.030

Silva, L. B. G., Rosado, E. L., Padilha, P. C., Dias, J. R., Moreira, T. M., Paula, T. P., Barros, D. C., \& Saunders, C. (2018). Food intake of women with gestational diabetes mellitus, in accordance with two methods of dietary guidance: a randomised controlled clinical trial. British Journal Of Nutrition, 121(1), 82-92. https://doi.org/10.1017/S0007114518001484

Silveira, L. R. P., Schmidt, M. I., Reichelt, A. A. J., \& Drehmer, M. (2020). Obesity, gestational weight gain, and birth weight in women with gestational diabetes: the linda-brasil (2014-2017) and the ebdg (1991-1995) studies. Jornal de Pediatria, S0021-7557(19), 30560-1. https://doi.org/10.1016/j.jped.2020.02.004

Sociedade Brasileira de Diabetes. (2019). Diretrizes da Sociedade Brasileira de Diabetes 2019-2020. Editora Clannad. https://www.diabetes.org.br/profissionais/images/DIRETRIZES-COMPLETA-2019-2020.pdf

Tavares, M. G. R., Lopes, E. S., Barros, R. A. J. P. A., Azulay, R. S. S., \& Faria, M. S. (2019). Profile of pregnant women with gestational diabetes mellitus at increased risk for large for gestational age newborns. Revista Brasileira de Ginecologia e Obstetrícia, 41(05), 298-305. https://doi.org/10.1055/s-00391687860

Trujillo, J., Vigo, A., Reichelt, A., Duncan, B. B., \& Schmidt, M. I. (2014). Fasting plasma glucose to avoid a full OGTT in the diagnosis of gestational diabetes. Diabetes research and clinical practice, 105(3), 322-326. https://doi.org/10.1016/j.diabres.2014.06.001

World Health Organization. (2000). Obesity: preventing and managing the global epidemic: Report of a World Health Organization consultation. https://apps.who.int/iris/handle/10665/42330

Yen, I. W., Lee, C. N., Lin, M. W., Fan, K. C., Wei, J. N., Chen, K. Y., Chen, S. C., Tai, Y., Kuo, C. H., \& Lin, C. H. (2019). Overweight and obesity are associated with clustering of metabolic risk factors in early pregnancy and the risk of GDM. Plos One, 14(12), 225-978.

https://doi.org/10.1371/journal.pone.0225978

Zaman, F., Nouhjah, S., Shahbazian, H., Shahbazian, N., Latifi, S. M., \& Jahanshahi, Al. (2018). Risk factors of gestational diabetes mellitus using results of a prospective population-based study in Iranian pregnant women. Diabetes \& Metabolic Syndrome: Clinical Research \& Reviews, 12(5), 721-725. https://doi.org/10.1016/j.dsx.2018.04.014 